\title{
Keck Planet Finder
}

Steven R. Gibsona, Andrew W. Howardb,a, Kodi Ridera, Arpita Royc, Jerry Edelsteina, Marc Kassis ${ }^{d}$, Jason Grilloa, Sam Halversone, Martin M. Sirka, Christopher Smitha, Steve Allen $f$, Ashley Baker ${ }^{b},{ }^{*}$, Charles Beichmang, Bruce Berrimang, Thomas Brown ${ }^{d}$, Kelleen Casey ${ }^{d}$ Jason Chin ${ }^{d}$, David Couttsh , David Cowleyf, William Deichf, Tobias Fegerh, Benjamin Fultong, Luke Gers, Yulia Gurevich', Yuzo Ishikawa',

Ean James ${ }^{d}$, Sharon Jelinsky ${ }^{a}$, Stephen Kaye ${ }^{b}$, Kyle Lanclos ${ }^{d}$, Adela Lia, Scott Lilleyd, Ben McCarney, ${ }^{d}$ Tim Millera, Steve Milner ${ }^{d}$,

Timothy J. O'Hanlon, Jacob Pember h, Mike Raffantia, Constance Rockosif,j, Ryan Rubenzahl ${ }^{b}$, Dave Rumph ${ }^{b}$, Dale Sandfordf,

SPACE SCIENCES LABORATORY MACQUARIE Berkeley Maureen Savagef, Christian Schwabh, Andreas Seifahrtk, Abby Shaumg, Roger Smith ${ }^{b}$, Julian Stuermerl, Jim Thorned, Adam Vandenbergd, Tod Von Boeckmann ${ }^{d}$, Cindy Wang, Qifan Wang ${ }^{b}$, Marie Weisfeiler ${ }^{\mathrm{a}, \mathrm{m}}$, Mavourneen Wilcox ${ }^{\mathrm{d}}$, Edward H. Wishnow ${ }^{\mathrm{a}}$, Peter Wizinowich ${ }^{\mathrm{d}}$, Truman Wold ${ }^{d}$ and Anna Wolfenberger ${ }^{a}$

Space Sciences Laboratory, University of California; 'CCalifornia Institute of Technology; 'Space Telescope Science Institute; 'W. M. Keck Observatory; et Propulsion Laboratory, California Institute of Technology; 'University of California Observatories; EIPAC-NASA Exoplanet Science Institute; ${ }^{~}$ Macquarie University, Johns Hopkins University; 'University of California, Santa Cruz; 'University of Chicago; 'Heidelberg University; 'mColumbia Law School; "51 Pegasi b Fellow

W. M. KECK OBSERVATORY

\section{Caltech}

UNIVERSITY OF CALIFORNIA OBSERVATORIES

\section{Introduction}

KPF is a fiber-fed, high-resolution, high-stability spectrometer in development for the W.M. Keck Observatory. KPF will characterize exoplanets via Doppler spectroscopy with a single measurement precision of $0.5 \mathrm{~m} \mathrm{~s}^{-1}$ (requirement) to $0.3 \mathrm{~m} \mathrm{~s}^{-1}$ (goal).

Fabrication of the majority of KPF subsystems is currently underway. Assembly of the main spectrometer will begin in early 2021.

\section{Optical Fiber Cables}

Optical fibers are protected by Miniflex PBT tubing. A strain relief box is placed at the mid-point of the $\sim 60 \mathrm{~m}$ fiber cables, to absorb relative movements between fiber and Miniflex.

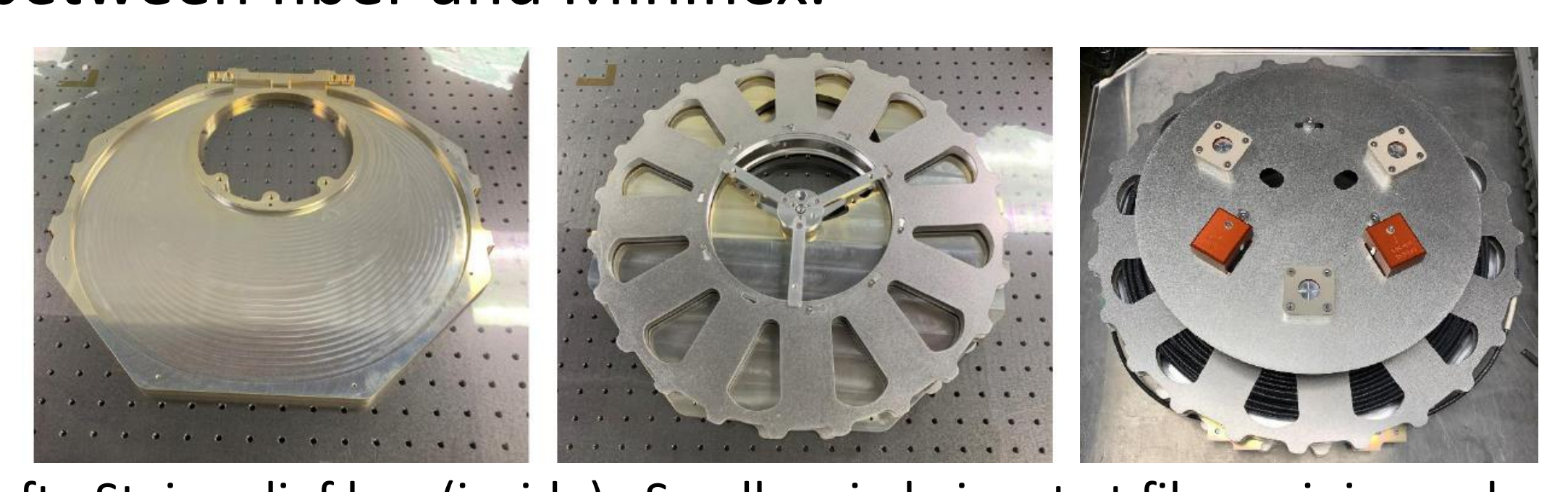

Left: Stain relief box (inside). Smaller circle is set at fiber minimum ben radius. Center: Miniflex storage spool mounted on strain relief box. Right: Strain relief box and spool in fiber AR-coating configuration

\section{Fiber Agitator}

- Suppresses modal noise within science, sky and simultaneous calibration fibers

- New design comprised of fiber within two $\varnothing 0.5 \mathrm{~m}$ loops of Miniflex PBT tubing.

- As motor-driven disk spins, the bars tap the fiber loops, which oscillate and resonate in three directions at several different frequencies.

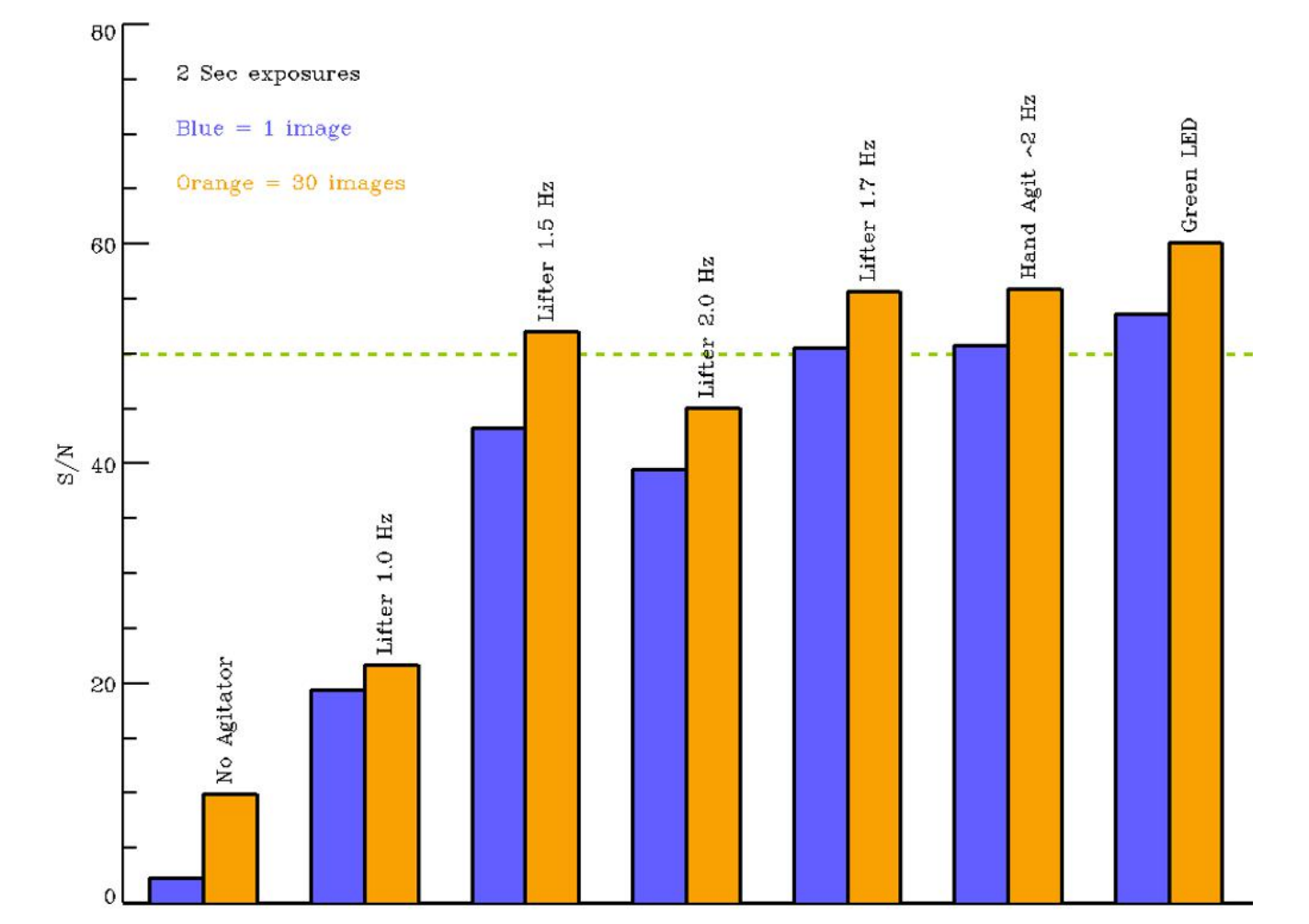

Lab tests with laser input light show SNR postagitation nearly equivalent to green LED.

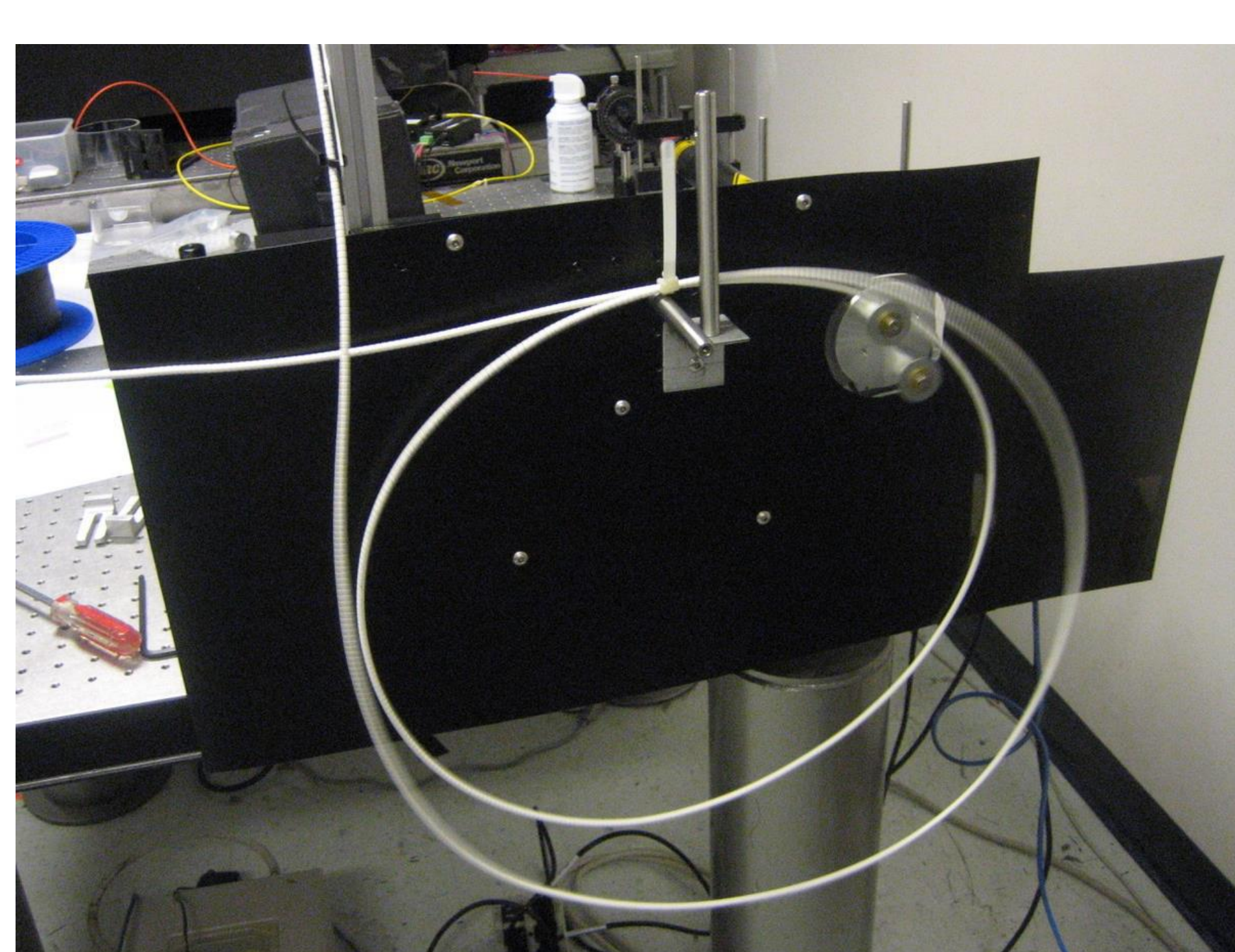

The KPF fiber agitator prototype in operation at $\sim 2 \mathrm{~Hz}$. Blurring of PBT tubing gives a sense of size of oscillations.
System Overview

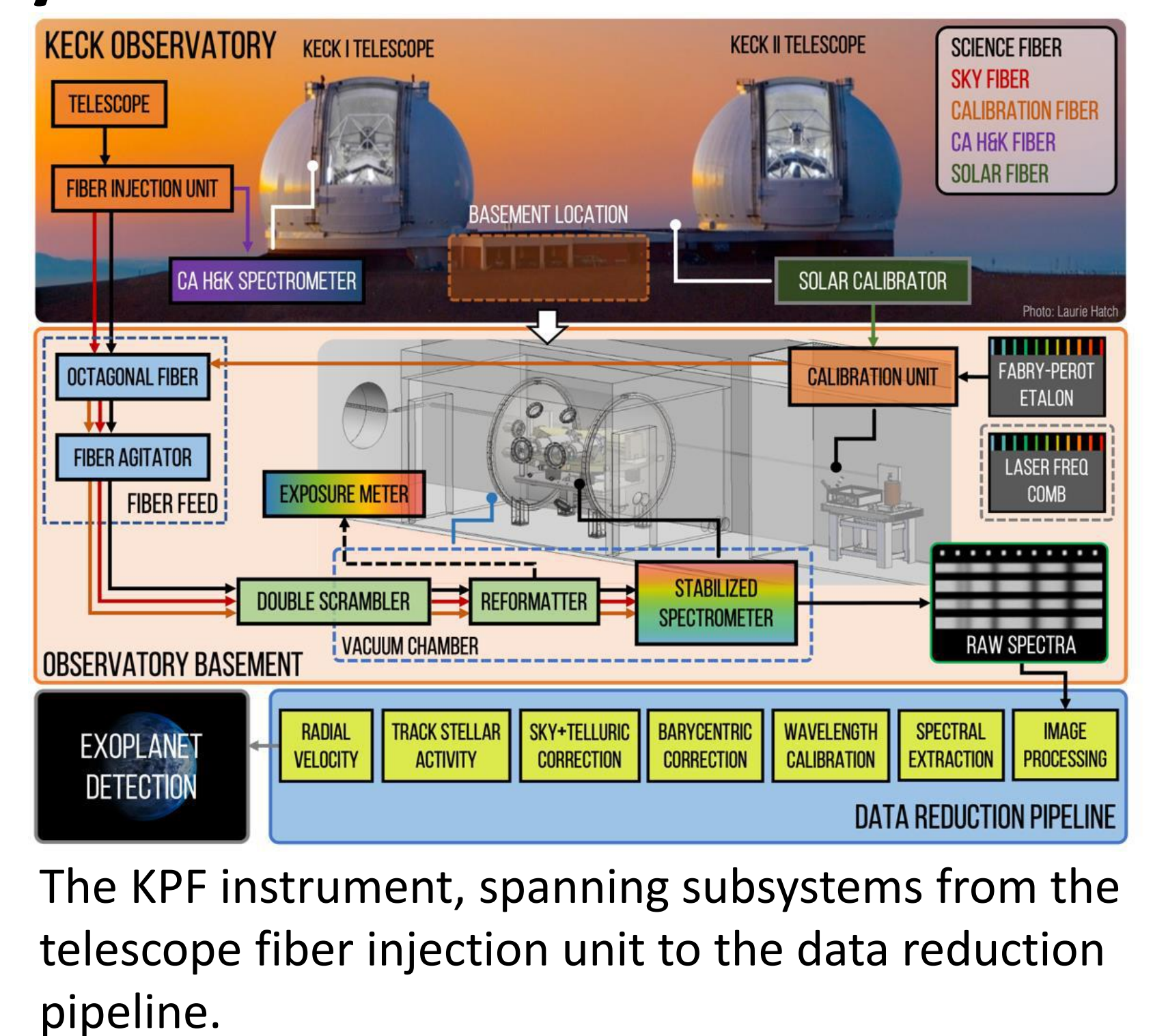

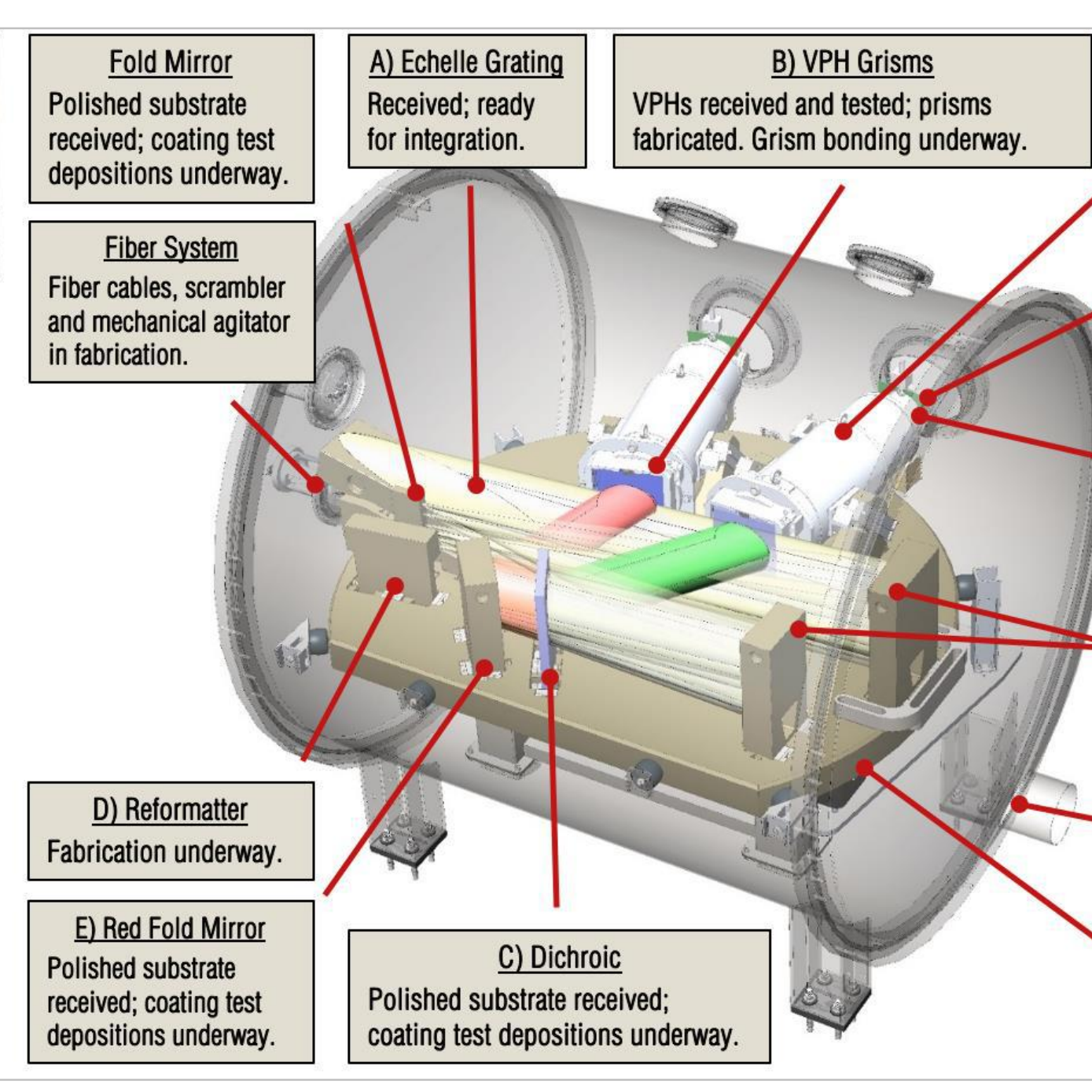

(nise

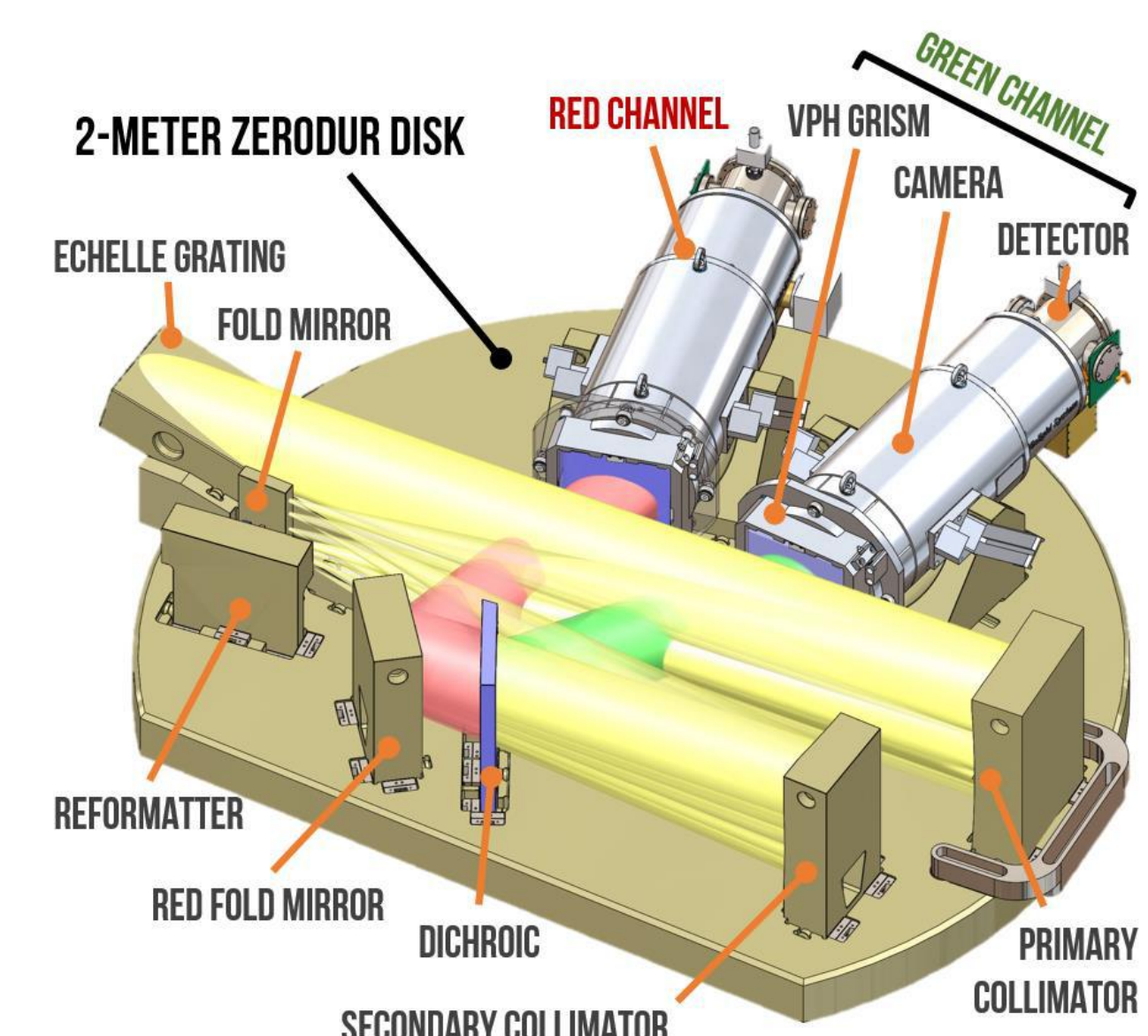

SEcondar CoLlumaroa

At the core is an asymmetric whitepupil spectrometer on a Zerodur be ur mounts and optics.

\section{Exposure Meter}

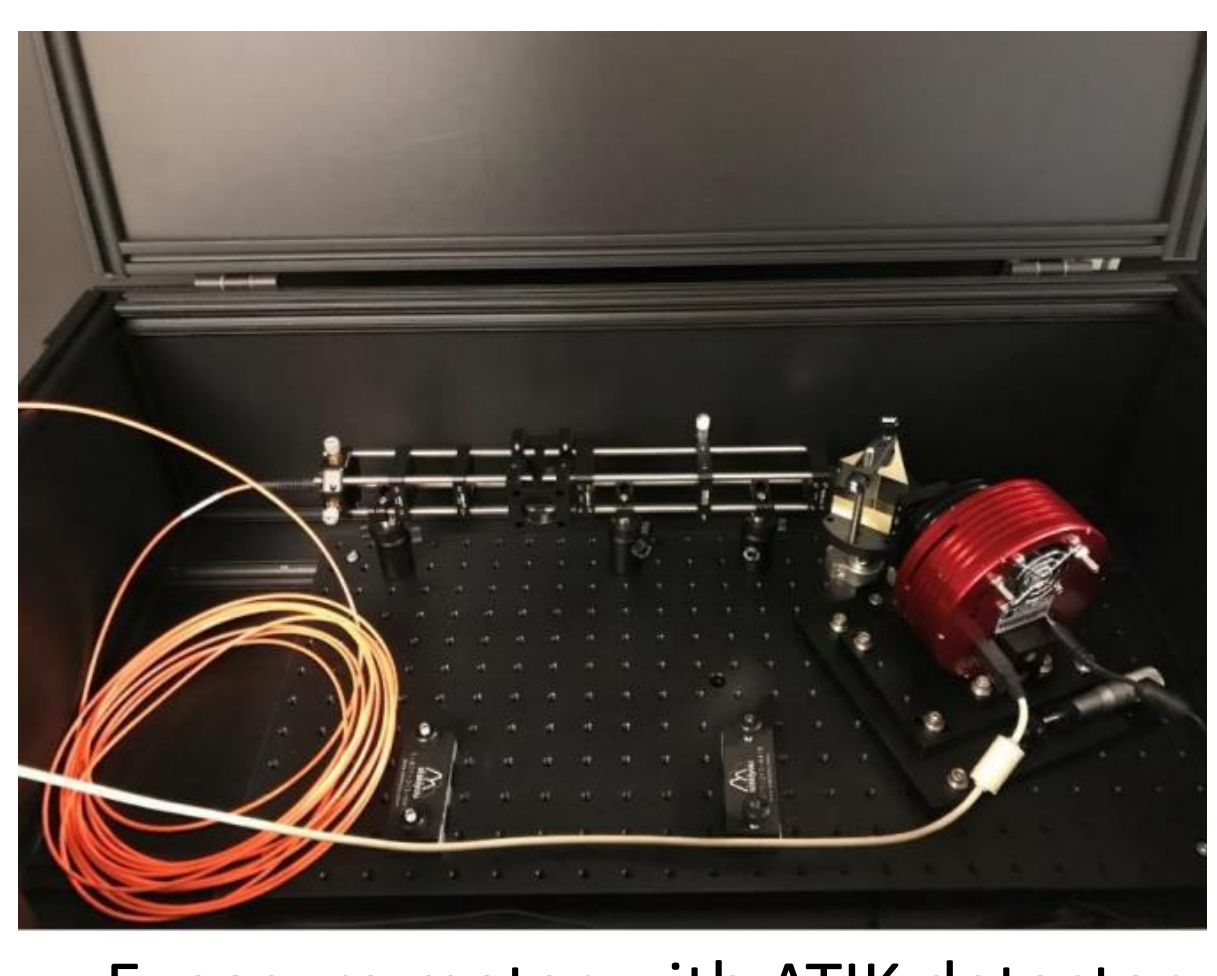

\section{Zerodur Bench Fabrication}

The KPF Zerodur disk has been sliced into sub-components at Corning. It is currently at Schott being prepared for final milling.

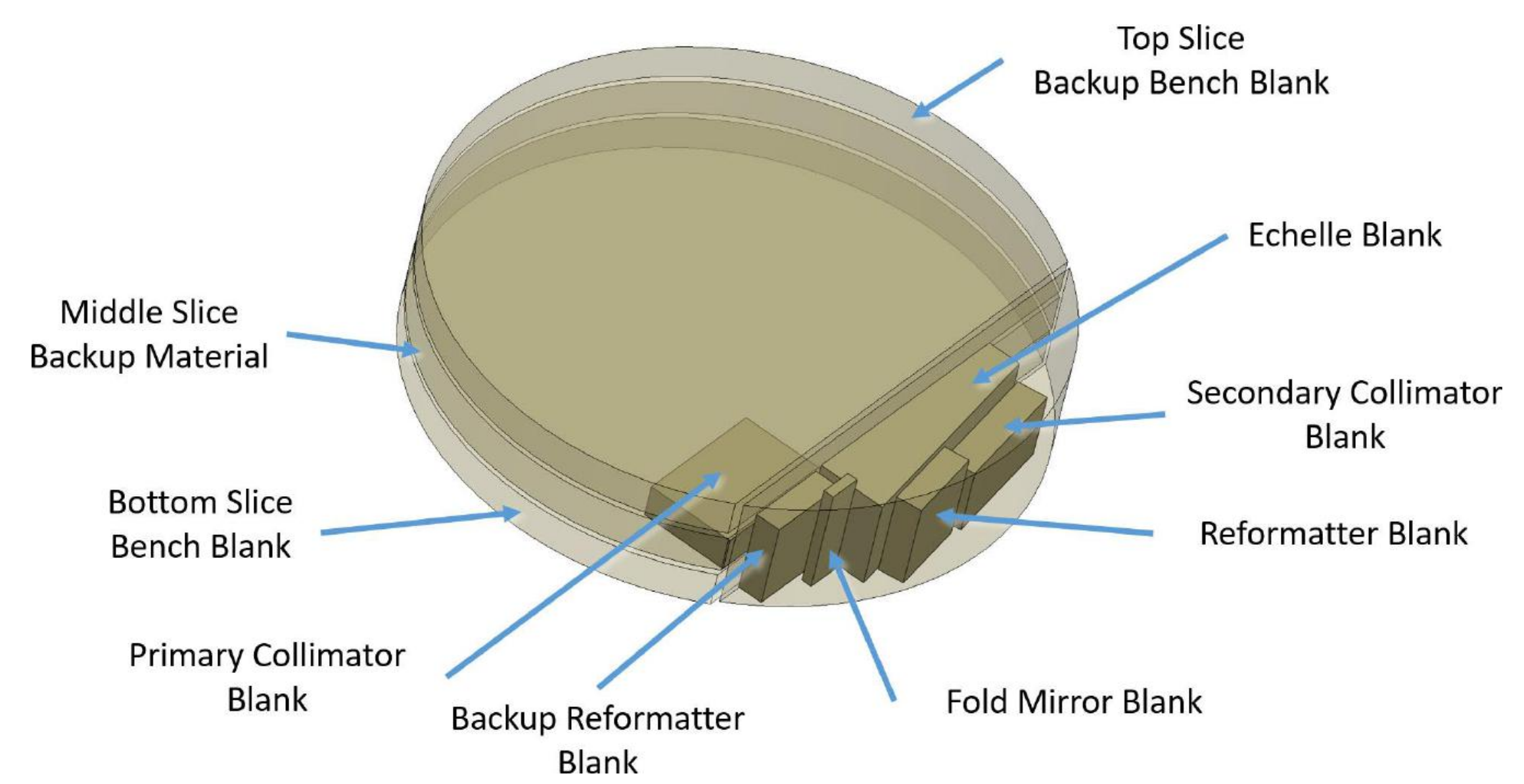

A Zerodur disk measuring $2 \mathrm{~m}$ diameter $\times 0.4 \mathrm{~m}$ thick supplied the $\mathrm{m}$

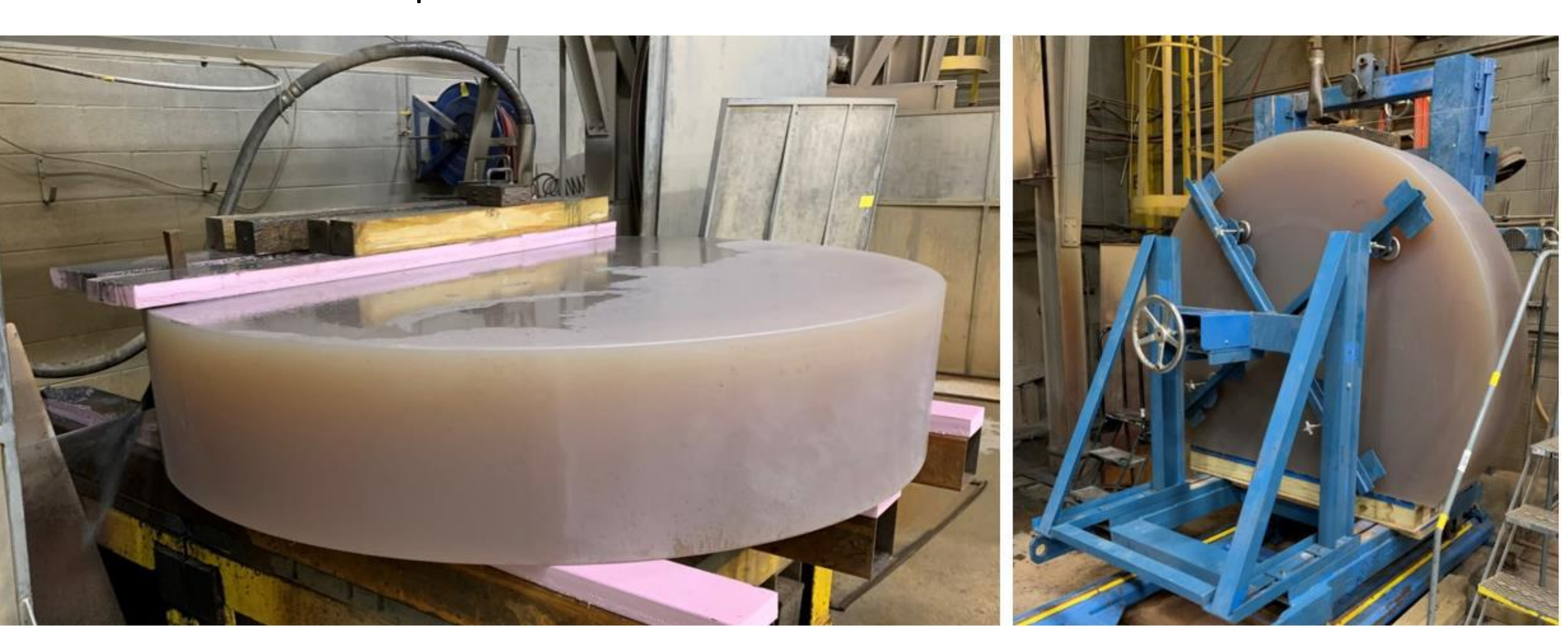

The KPF Zerodur disk on a wire saw at Corning. Left: D-Cut slice. Right: Bench slicing.

\section{Double-Fiber Scrambler}

- A double-fiber scrambler is utilized to stabilize and homogenize the illumination of the main spectrometer

- Optical and mechanical parts have been fabricated and final assembly is underway.

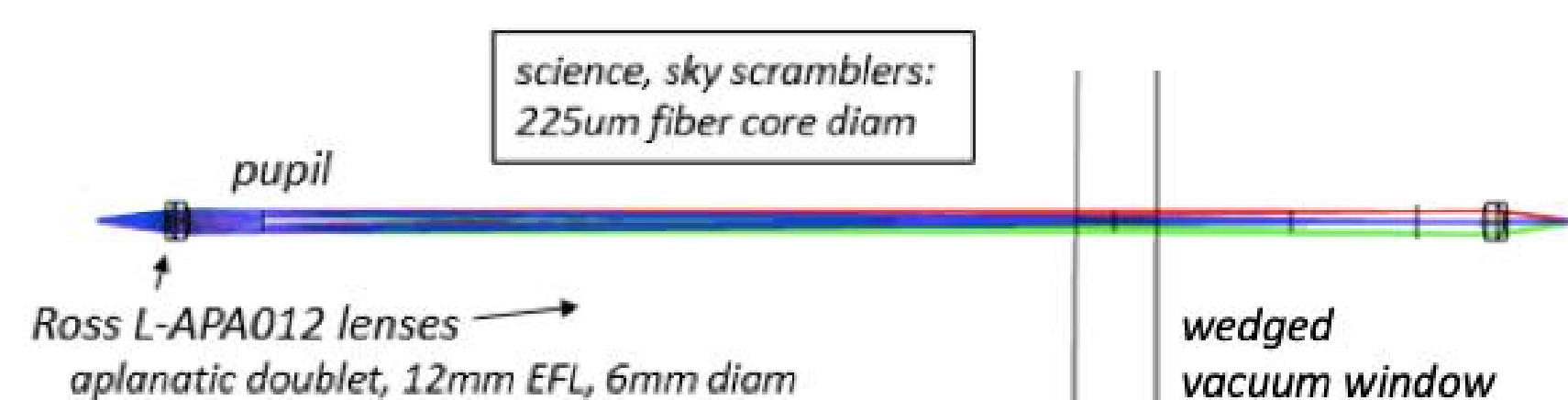

- Prism-based spectrometer $(R \sim 100)$ allows chromatic barycentric corrections

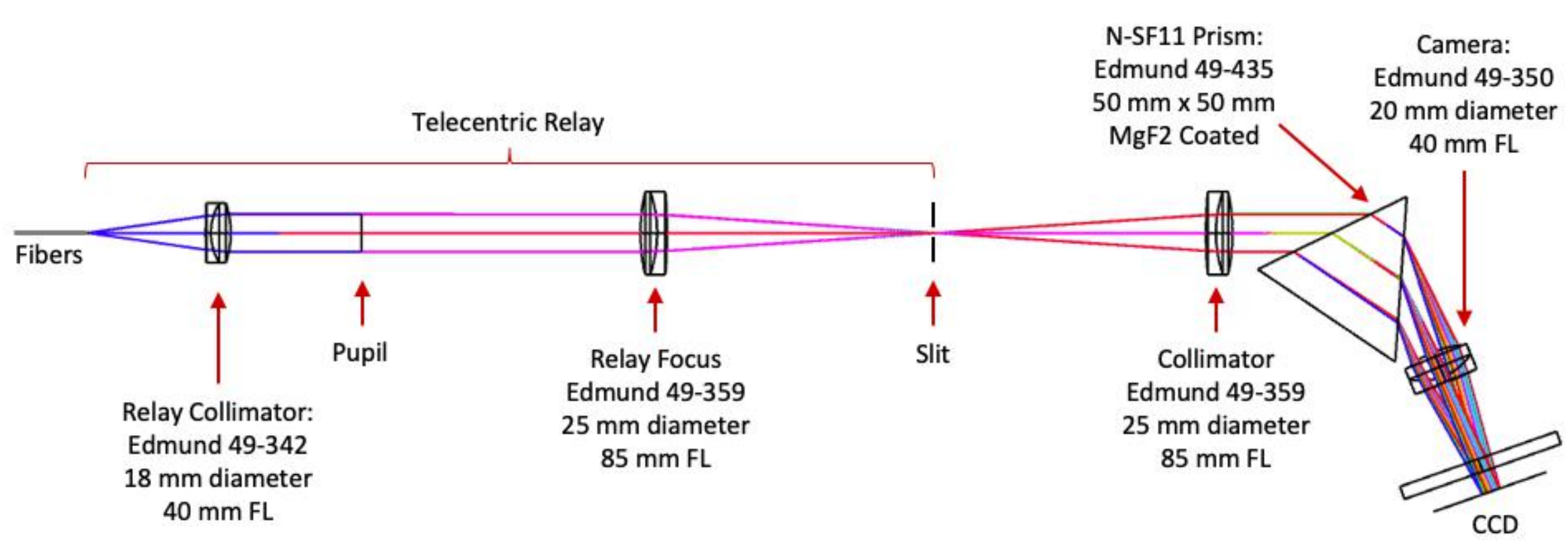

Optical design of the KPF exposure meter. Collimated beam diameter is $12 \mathrm{~mm}$.

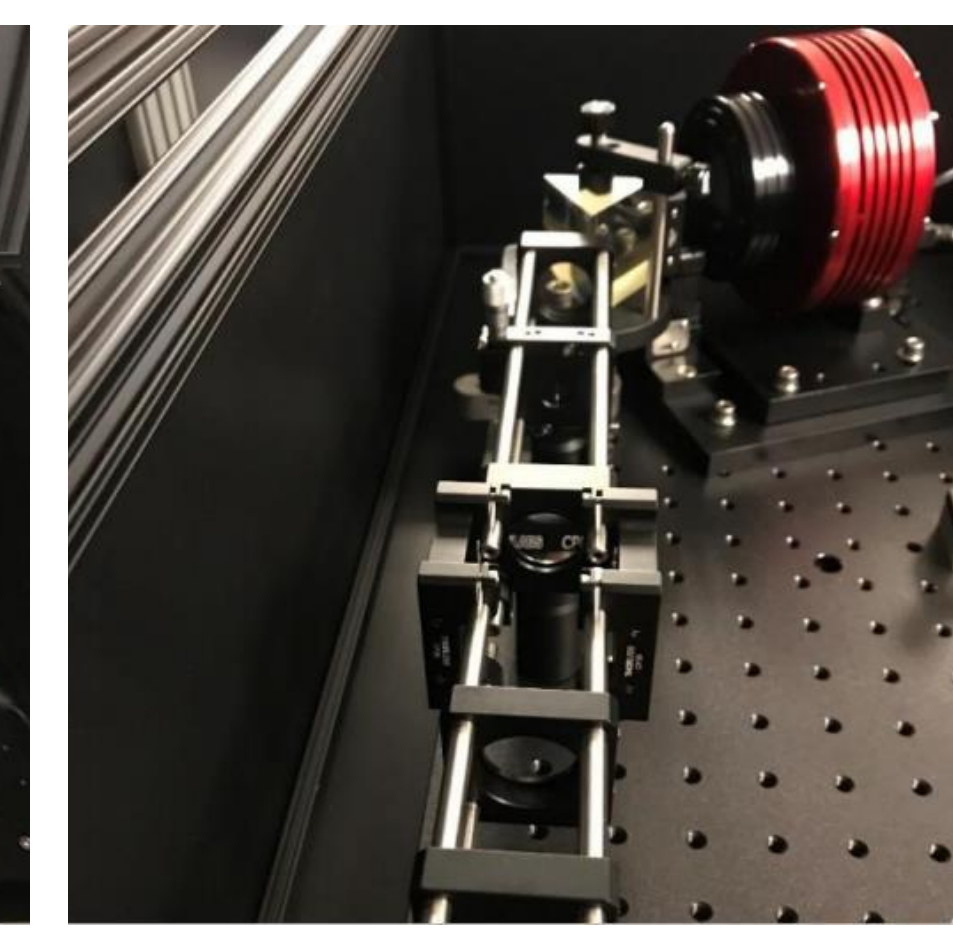

Exposure meter with ATIK detector used for preliminary testing.

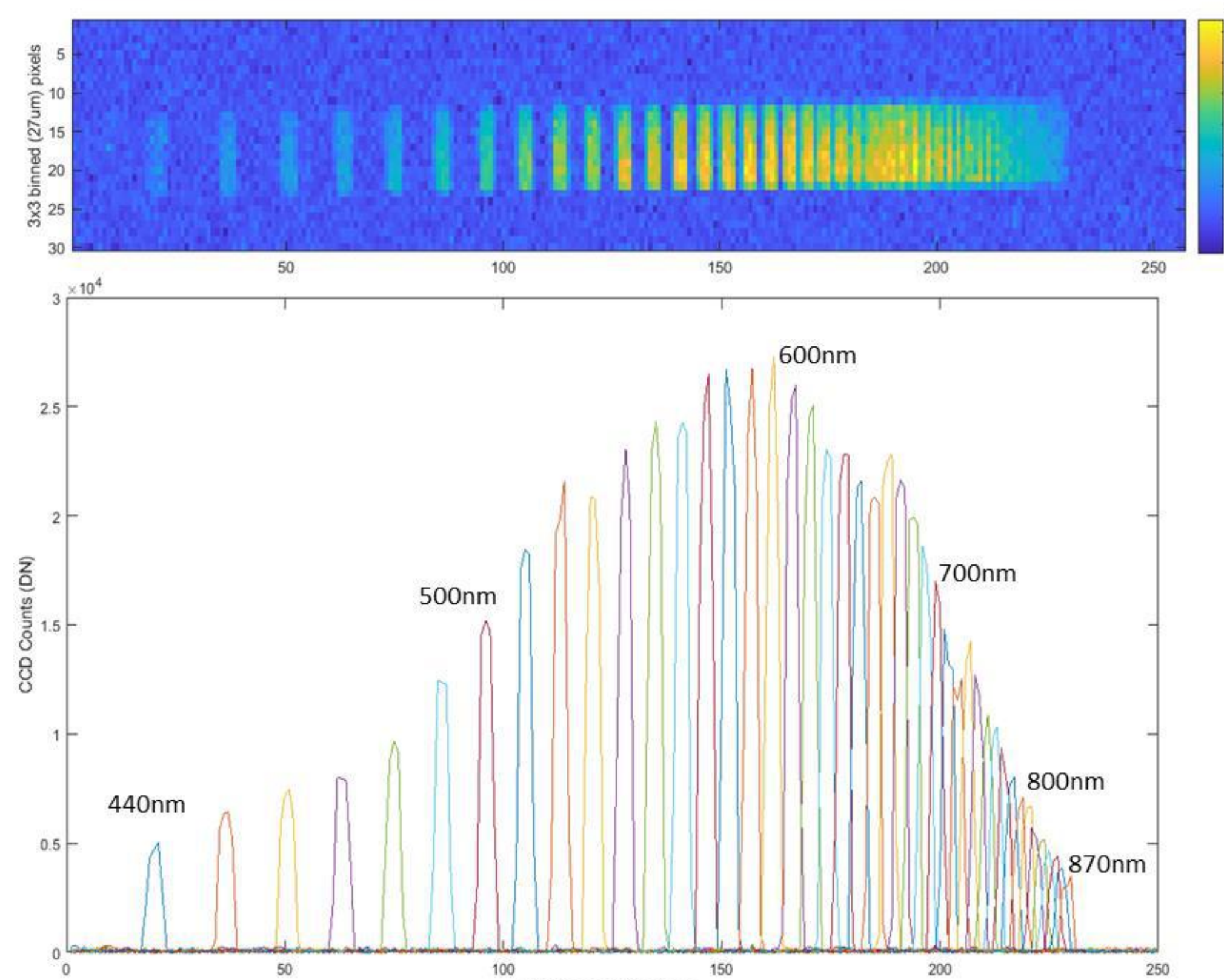

Co-added image of an exposure meter wavelength scan, using a monochromator as the source. The redward falloff is dominated by the ATIK 11000 detector used for preliminary lab tests.

bove: Optical layout. A vacuum window is placed in the air space between the two lenses, allowing the scrambler to act as an optical feedthrough for
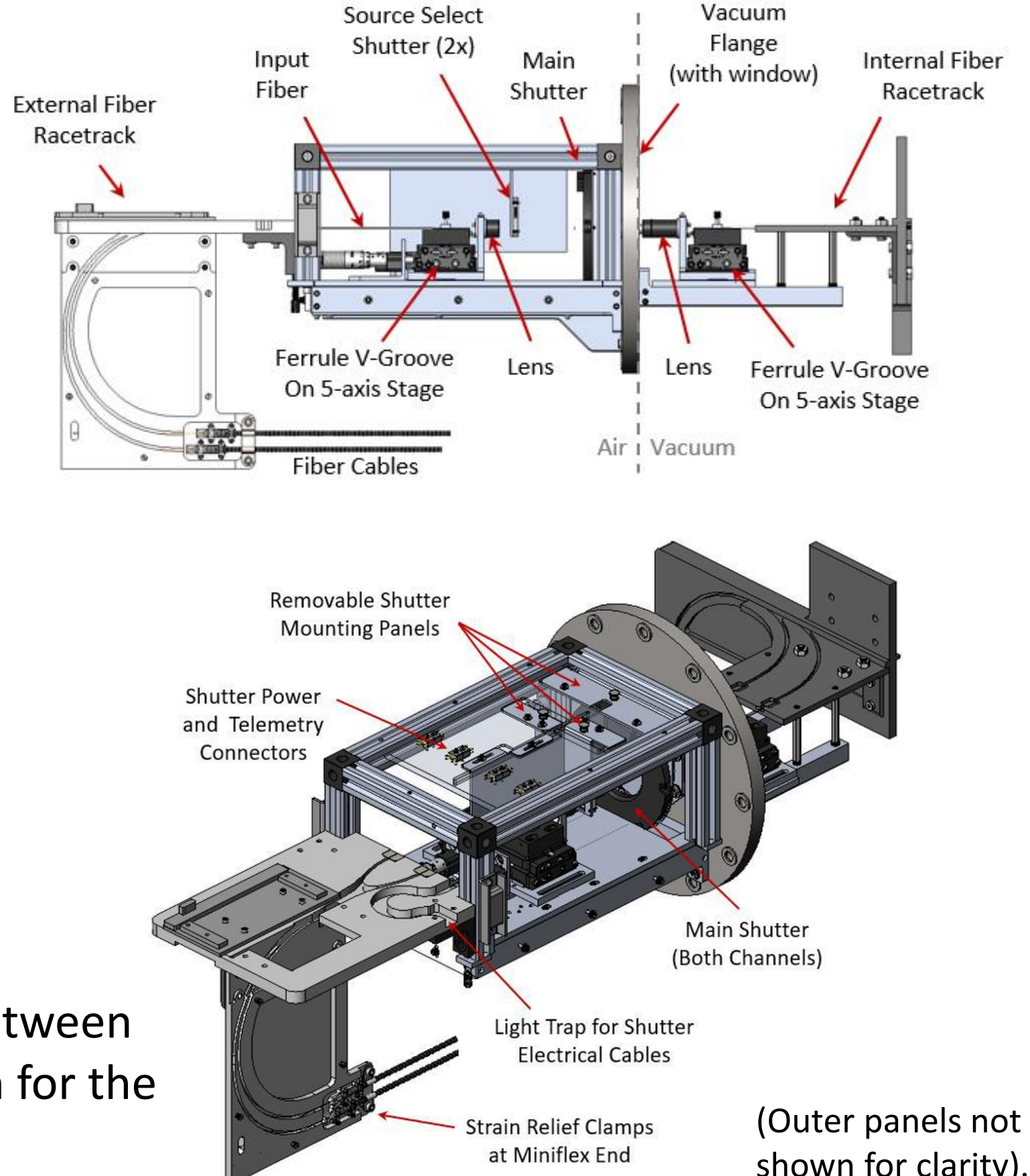

\section{VPH Grating Testing}

- Green and Red VPH gratings fabricated by Kaiser Optical Systems

- Grating throughput measured by KPF team

- Measured throughput exceeds expected 'production minimum'

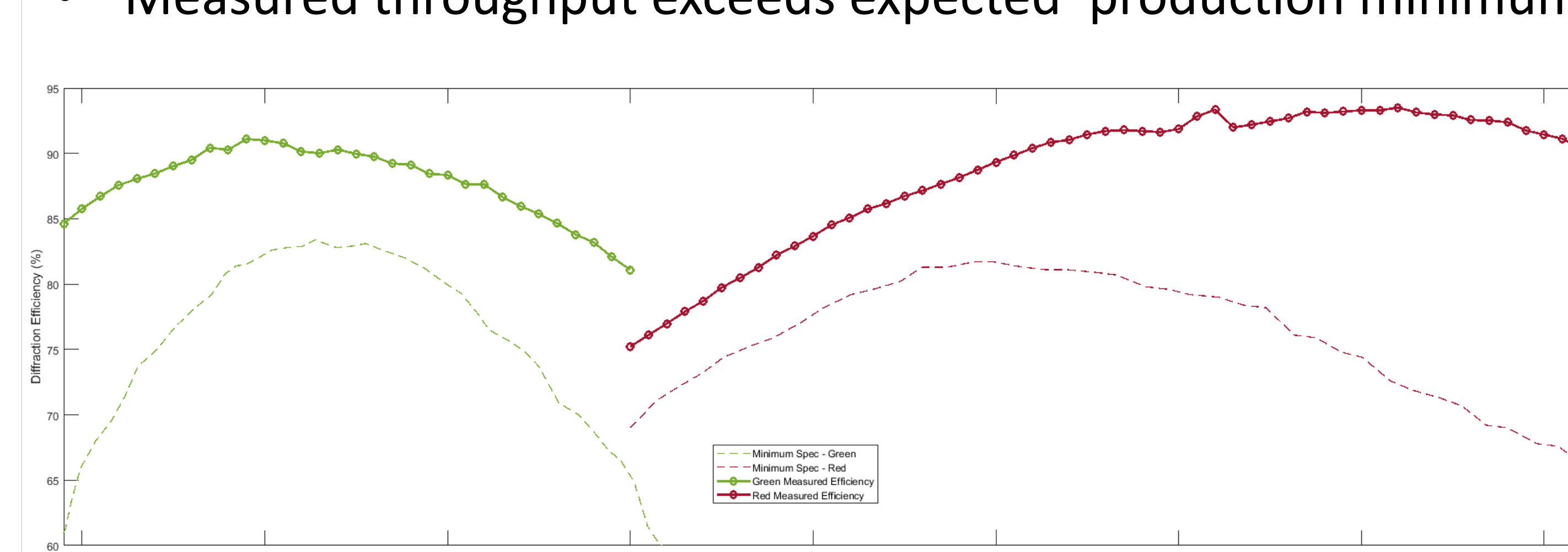

KPF VPH grating measurement results.

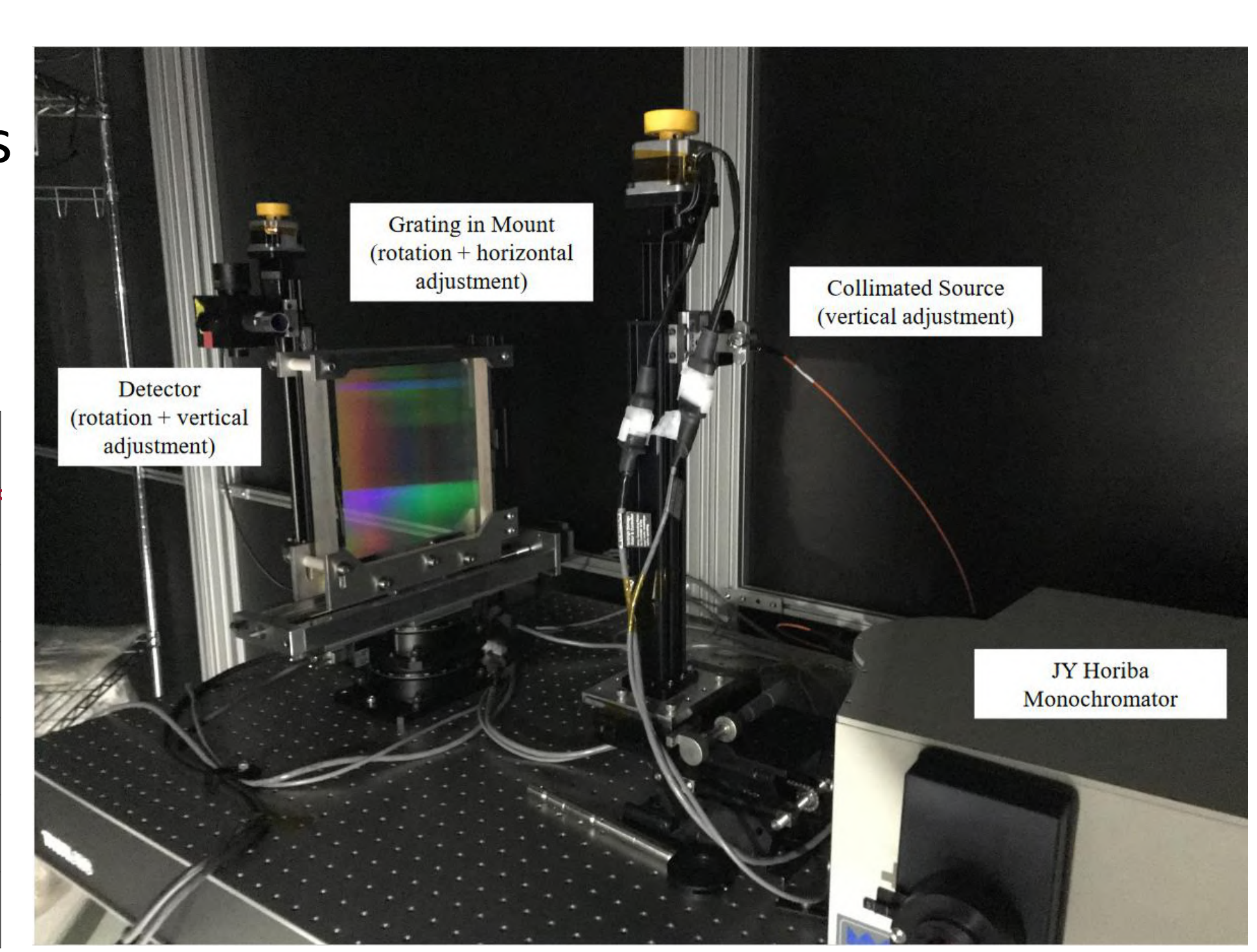

KPF VPH grating under test.

The authors thank the Heising-Simons Foundation, the National Science Foundation (award 2034278 through the Mid-Scale Innovations Program in Astronomical Sciences) private donors, the W.M. Keck Foundation, the University of California, Berkeley, the California Institute of Technology, the University of Hawaii, the Jet Propulsion Laboratory, and the Mt. Cuba Astronomical Foundation for financial support of KPF. The authors also wish to thank Winlight Systems for their valuable contributions to the designs of the 